\title{
Exploring scenarios of dietary exposure to estimate the intake of Sucralose by young children with medical conditions
}

\author{
A.J. O’Sullivan and A.I. McKevitt \\ Institute of Food and Health, University College Dublin, Belfield, Dublin 4, Ireland.
}

Certain medical conditions e.g. inborn errors of metabolism (Phenylketonuria, PKU) or severe cow's milk protein allergy (CMPA) require dietary modification in order that clinical symptoms can be managed. Specially formulated medical nutrition products have been developed to manage the dietary restrictions imposed on patients with these conditions e.g. the partial replacement of high protein foods or cow's milk protein in the diet.

The artificial sweetener Sucralose (E 955) is used to improve the appearance and palatability of these medical nutrition products. Young children have a higher risk of exceeding the acceptable daily intake (ADI) for food additives than adults due to higher food intake per $\mathrm{kg}$ body weight ${ }^{(1,2,3)}$.

The aim of this project was to use dietary exposure modelling to explore different scenarios of Sucralose intake by young PKU and CMPA patients (1-3 years) from the UK.

Food consumption data for patients with PKU and CMPA are not readily available. Therefore food consumption data for healthy young children were used as a baseline for analysis The National Diet and Nutrition Survey (NDNS) 2008-2012 was designed to assess the diet, nutrient intake and nutritional status of the healthy UK children aged 1.5-4.5 years. The population of interest was young children aged from 1-3 years (386 subjects). Food groups used for data collection in this study are the 17 EU food categories. An adapted validated probabilistic model was used to assess patients' exposure to Sucralose ${ }^{(4,5,6)}$. Medical nutrition products were included in the exposure model to replace restricted foods. This replacement was based on recommendations for adequate protein intake and dietary adherence data.

Exposure assessment results indicate young UK patients with PKU and CMPA have higher relative average intakes of Sucralose than healthy young children (see tables).

Table 1. Additive exposure estimates for young patients with PKU

\begin{tabular}{lccrc}
\hline & \multicolumn{4}{c}{ PKU $(\mathrm{mg} / \mathrm{kg} / \mathrm{d})$} \\
\cline { 2 - 5 } & Mean & SE & P95 & SE \\
\hline Baseline & 2.10 & 0.33 & 7.48 & 0.76 \\
Scenario 1 (moderate adherence) & 6.22 & 0.14 & 12.05 & 0.55 \\
Scenario 2 (high adherence) & 8.34 & 0.14 & 16.93 & 0.55 \\
\hline
\end{tabular}

Table 2. Additive exposure estimates for young patients with CMPA

\begin{tabular}{lccrr}
\hline & \multicolumn{3}{c}{ CMPA $(\mathrm{mg} / \mathrm{kg} / \mathrm{d})$} \\
\cline { 2 - 5 } & Mean & SE & P95 & SE \\
\hline Baseline & 2.05 & $0 \cdot 38$ & $7 \cdot 11$ & 1.37 \\
Scenario 1 (moderate adherence) & 4.25 & 0.22 & 9.57 & $0 \cdot 81$ \\
Scenario 2 (high adherence) & 6.36 & 0.22 & 14.50 & 0.81 \\
\hline
\end{tabular}

Two replacement scenarios of dietary adherence were explored: High adherence (75\% replacement) and moderate adherence ( $50 \%)$. Based on modelled exposures, certain high consumers (P95) with PKU may exceed the established ADI for Sucralose (15 mg/kg/day) in the high adherence scenario but not in the moderate adherence scenario. For CMPA, the established ADI was not exceeded for Sucralose. The results underline the necessity to use Medical nutrition-relevant models in the estimation of patient exposures. Dietary exposure modelling provides for the estimation of dietary exposure to Sucralose in patients, which would otherwise not be possible

Work supported by Danone Trading Medical B.V. Schiphol Boulevard 105, 1118 BG Schiphol Airport, The Netherlands

1. Bearer CF (1995) Future Child 5, 11-26.

2. Lawrie C (1998) Food Add Contam 15, 75-81.

3. VWA (2008) Report of a VWA panel. The Hague, The Netherlands.

4. McNamara C, Naddy B, Rohan D, Sexton J (2003) Food Addit Contam. 20:S8-S26.

5. Gilsenan MB, Lambe J, Gibney MJ 2002. Food Addit Contam. 19:1105-1115.

6. Lambe J (2002) Proc Nutr Soc. 61, 11-18. 